\title{
Spore formation in plants: SPOROCYTELESS and more
}

\author{
Cell Research (2015) 25:7-8. doi:10.1038/cr.2014.164; published online 16 December 2014
}

Plant reproduction is initiated by the specification of sporocytes that form haploid spores through meiosis. A new study in Arabidopsis published in Cell Research shows how the product of SPOROCYTELESS/NOZZLE, a key gene in this process, partners with co-repressors and transcription factors to promote spore formation, and draws interesting parallels with fungi.

The life cycle of plants alternates between a diploid sporophytic generation and a haploid gametophytic generation. The transition from sporophyte to gametophyte requires the formation of haploid cells (spores) by meiosis, a process called sporogenesis. In flowering plants, sporogenesis is initiated in both male and female reproductive tissues by the differentiation of sub-epidermal cells in the anther and ovule primordia, called archesporial cells (ACs), into premeiotic cells called sporocytes. Both male sporocytes (microsporocytes) and female sporocytes (megasporocytes) will then undergo meiosis to give rise to the microspores and megaspores, in the male and female organs, respectively. The specification of the sporocytes represents a critical step in the process of sporogenesis. The SPOROCYTELESS/NOZZLE (SPL/NZZ) gene which encodes a nuclear protein was identified as a key regulator of this stage of sporogenesis in Arabdiopsis [1, 2]. In $\mathrm{spl} / \mathrm{nzz}$ mutants, development of both male and female reproductive organs proceeds normally until the ACs are formed, but the ACs fail to differentiate into sporocytes. Subsequently, $S P L$ was found to be the target of regulation by the floral homeotic gene AGAMOUS $(A G)[3] . A G$ is a floral organ identity gene required for the specification of stamens and carpels in the third and fourth whorls of the flower [4]. $A G$ encodes a transcription factor of the MADS-box family expressed in stamen and carpel primordia, and is required for the activation of SPL expression [3]. In anthers, there are additionally two leucine-rich repeat receptor-like protein kinases, BARELY ANY MERISTEMI (BAM1) and BAM2, that act in a regulatory loop with $S P L$ to promote somatic cell types and to restrict $S P L$ expression to the inner region of the locule [5].

Despite the identification of $S P L /$ NZZ 15 years ago, and its central role in promoting the reproductive transition to the gametophytic generation, the molecular basis of gene regulation by $S P L$ has remained unknown. A new study by Wei et al. [6] has now revealed the molecular mechanism by which $S P L$ acts to control gene expression. Wei et al. show that the C-terminal end of SPL protein contains an EAR motif, which presents in many transcriptional repressors, and that an intact EAR motif is required for rescue of a $s p l$ mutation. Furthermore, SPL interacts with co-repressors of the TPL/TPR family through its EAR motif. Expression of a mutant protein SPLmEAR (SPL with mutated EAR motif) results in a $s p l$ mutant phenotype due to competition with endogenous SPL. This dominant negative mutant genetically interacts with the tpl-1 mutant which is defective for the TPL1 gene. Moreover, in tpl-1 homozygous mutant ovules, ACs failed to differentiate into megasporocytes. These results suggest that SPL regulates megasprogenesis through the recruitment of the TPL1 co-repressor, forming a repressor complex.

Which genes might be the target of regulation by this repressor complex? By using yeast 2-hybrid screens, Wei et al. show that the N-terminus of SPL interacts with transcription factors of the TCP (TEOSINTE BRANCHEDI/ CYCLOIDEA/PROLIFERATING CELL FACTOR) family [7]. Moreover, overexpression of $S P L$ resulted in phenotypes resembling loss-of-function mutants in the class II TCP genes that are required for leaf development, suggesting that ectopic SPL interferes with TCP functions in the leaf. Wei et al. also found that class II TCPS are expressed in the ovule, consistent with their roles in ovule development. Overexpression of these TCPS results in ovule phenotypes similar to $s p l$ mutants, including suppression of $\mathrm{AC}$ differentiation into megasporocytes. Furthermore, proteinprotein interaction experiments showed that SPL likely serves as a bridge between TCPs and TPL/TPR proteins. In shoot meristems, the $C U C$ (Cup Shaped Cotyledon) genes that promote meristem formation are the presumptive targets of class II TCPs. Class II TCPs in the shoot act to restrict undifferentiated or meristematic cell fate through downregulation of $C U C$ genes at organ boundaries, and thus promote organ differentiation [8]. The target genes of $T C P s$ in ovules and anthers are yet to be identified, but may have a similar role to those in shoots in preventing differentiation. The findings by Wei $e t$ $a l$. provide for the first time insights into the molecular mechanism for the initiation of sporogenesis in a flowering plant (Figure 1). The floral homeotic gene $A G$ activates $S P L$ expression in the arche- 


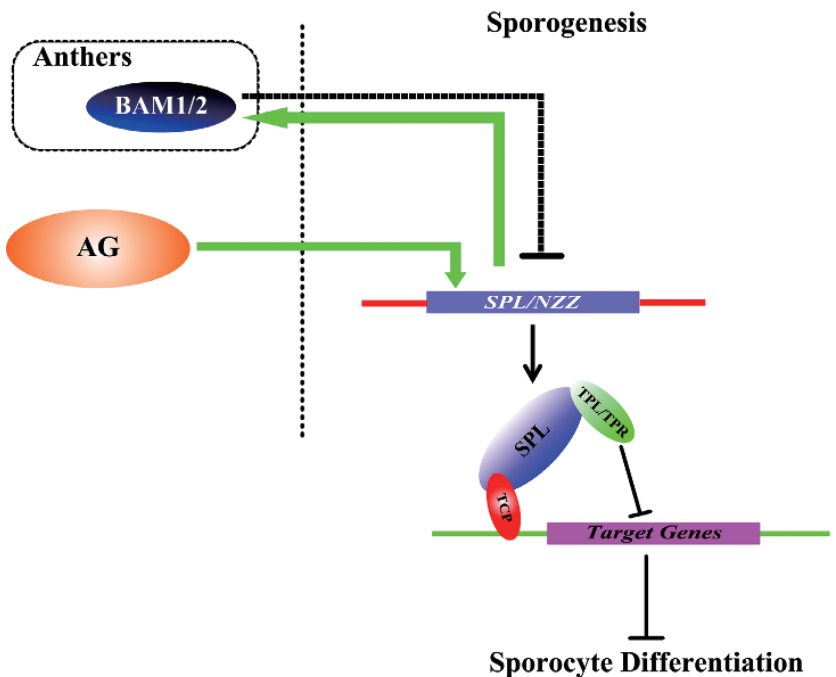

Figure 1 Sporogenesis in Arabidopsis. Schematic representation, based on the results of Wei et al., and incorporating the previously known genetic interactions between genes involved in sporogenesis in Arabidopsis. Arrows indicate positive regulation, and black T-bars indicate negative regulation. Dotted lines show indirect interactions.

sporial cells of anthers and ovules. SPL protein associates with class II TCPs and recruits TPL/TPR co-repressors to suppress target genes of the TCP transcription factors. It can be concluded that $S P L$ likely promotes sporogenesis by acting in concert with TCPs and TPL/ TPRs to suppress negative regulators of sporocyte differentiation.

This proposed mechanism for regulating sporogenesis in Arabidopsis shows some remarkable parallels to that in fungi. Similar to plants, fungi commonly have alternating haploid and diploid generations, and the formation of haploid spores constitutes an important step in their reproductive strategies. The TPL/TPR co-repressors are part of the larger family of Groucho/Tup1 co-repressor proteins (named after the Drosophila Groucho and yeast Tup1 proteins) that are found in animals, plants and fungi [9]. It has been shown that the disruption of Tup1 in yeast and its orthologs in some fungal pathogens, leads to defective spore production [10]. Most interestingly, in yeast there is a protein SSN6 that serves as an adaptor for the suppressor activity [11], similar to the role of SPL in Arabidopsis. The similarities between yeast and Arabidopsis in mechanisms governing a process that is as basic as reproduction raise the interesting question of whether this mechanism might be ancestral, possibly reflecting an original function of these co-repressors in promoting spore formation that has been retained in both the plant and fungal kingdoms.

\section{Li Yuan ${ }^{1}$, Venkatesan Sundaresan ${ }^{1,2}$ \\ Department of ${ }^{1}$ Plant Biology and ${ }^{2}$ Plant Sci- ences, University of California-Davis, Davis, CA 95616, USA \\ Correspondence: Venkatesan Sundaresan \\ E-mail: sundar@ucdavis.edu}

\section{References}

1 Yang WC, Ye D, Xu J, et al. Genes Dev 1999; 13:2108-2117.

2 Schiefthaler U, Balasubramanian S, Sieber P, et al. Proc Natl Acad Sci USA 1999; 96:11664-11669.

3 Ito T, Wellmer F, Yu H, et al. Nature 2004; 430:356-360.

4 Bowman JL, Smyth DR, Meyerowitz EM. Development 1991; 112:1-20.

5 Hord CL, Chen C, DeYoung BJ, et al. Plant Cell 2006; 18:1667-1680.

6 Wei B, Zhang J, Pang C, et al. Cell Res 2015; 25:121-134.

7 Martín-Trillo M, Cubas P. Trends Plant Sci 2010; 15:31-39.

8 Koyama T, Furutani M, Tasaka M, et al. Plant Cell 2007; 19:473-484.

9 Lee JE, Golz JF. Plant Signal Behav 2012; 7:86-92.

10 Elías-Villalobos A, Fernández-Álvarez A, Ibeas JI. PLoS Pathog 2011; 7:e1002235.

11 Friesen H, Hepworth SR, Segall J. Mol Cell Biol 1997; 17:123-134. 